\title{
Efecto de dos maniobras cíclicas de reclutamiento alveolar sobre diversos parámetros de monitoreo microcirculatorio no invasivo en perros
}

\section{Effect of two cyclical alveolar recruitment maneuvers on different parameters of non-invasive microcirculatory monitoring in dogs}

\author{
Edwin Buriticá Gaviria'; Diego Echeverry Bonilla²; Diego Ospina Argüelles
}

\begin{abstract}
${ }^{1} M V Z$, Esp. Docencia universitaria, Esp. Clínico en pequeñas especies animales, M.Sc Ciencias Veterinarias, Grupo de Investigación en Medicina y Cirugía de Pequeños Animales. Universidad del Tolima, Código postal 730006299, Ibagué - Tolima, Colombia, e-mail: efburiticag@ut.edu.co, (1Dhttp://orcid.org/0000-0002-7979-4135

${ }^{2}$ MVZ, Esp. Docencia universitaria, M.Sc. Técnicas de diagnóstico animal, Ph.D. Veterinaria., Grupo de Investigación en Medicina y Cirugía de Pequeños Animales. Universidad del Tolima, Ibagué - Tolima, Colombia, e-mail: decheverry@ut.edu.co, Dhttp://orcid.org/0000-0003-2005-5116
\end{abstract}

${ }^{3}$ MVZ, Esp. Pedagogía, Grupo de Investigación en Medicina y Cirugía de Pequeños Animales. Universidad del Tolima, Ibagué - Tolima, Colombia, e-mail: daospina@ut.edu.co, Dhttp://orcid.org/0000-0003-1778-1504

Cómo citar: Buriticá Gaviria, E.; Echeverry Bonilla, D.; Ospina Argüelles, D. 2019. Efecto de dos maniobras cíclicas de reclutamiento alveolar sobre diversos parámetros de monitoreo microcirculatorio no invasivo en perros. Rev. U.D.C.A Act. \& Div. Cient. 22(1):e1150. https://doi.org/10.31910/rudca.v22.n1.2019.1150

Artículo de acceso abierto publicado por Revista U.D.C.A Actualidad \& Divulgación Científica bajo una licencia Creative Commons CC BY-NC 4.0

Recibido: Julio 17 de 2017

Aceptado: Enero 22 de 2019

\section{RESUMEN}

Las maniobras de reclutamiento alveolar (MRA) son importantes para revertir la formación de atelectasias perioperatorias; no obstante, su realización está sujeta a cambios hemodinámicos significativos. El presente estudio evaluó el efecto de dos protocolos cíclicos de reclutamiento alveolar, mediante el incremento de la presión positiva al final de la espiración (PEEP), sobre tres parámetros de monitoreo, a saber: el gradiente de temperatura centro periférico (GT), el tiempo de relleno capilar (TRC) y la oximetría de pulso $\left(\mathrm{SpO}_{2}\right)$, en 28 perros anestesiados. Los animales, se asignaron aleatoriamente en tres grupos, según la MRA a realizar, así: grupo 1, protocolo corto (PC) reclutado con PEEP de $10 \mathrm{cmH}_{2} \mathrm{O}$; grupo 2, protocolo largo (PL) reclutado con PEEP de $20 \mathrm{mH}_{2} \mathrm{O}$ y grupo 3, protocolo control (PCo) ventilado con PEEP de $0 \mathrm{cmH}_{2} \mathrm{O}$. Los parámetros de monitoreo fueron evaluados para cada maniobra en cuatro tiempos: T1 (basal en pre-anestesia), T2 (pre-reclutamiento), T3 (30min post-maniobra) y T4 (60 min post-maniobra). En el T2, los valores de TRC presentaron una disminución significativa $(\mathrm{p}<0,05)$, respecto al tiempo basal. Posteriormente, en el T3, se evidenció una disminución estadísticamente significativa del GT para el grupo PC frente a los demás tratamientos; en el T4, el TRC presentó una disminución estadísticamente significativa $(p<0,05)$ para el PL frente a los demás tratamientos. Se sugiere que, de acuerdo con los resultados encontrados, el protocolo de ventilación con incrementos de PEEP de hasta $20 \mathrm{cmH}_{2} \mathrm{O}$ es la MRA de elección, al no producir alteraciones de importancia clínica en los parámetros microcirculatorios evaluados.

Palabras clave: anestesia; atelectasia pulmonar; ventilación mecánica; monitoreo anestésico. 


\section{ABSTRACT}

Alveolar recruitment maneuvers (ARM) are important in reversing the formation of perioperative atelectasis; however, its performance is subject to significant hemodynamic changes. The present study evaluated the effect of two cyclic alveolar recruitment protocols, by increasing positive end-expiratory pressure (PEEP), on three parameters of microcirculatory monitoring in 28 anesthetized dogs. Animals were randomly assigned to one of three groups, according to the ARM to be performed, thus: Group 1, short protocol (SP) recruited with PEEP of $10 \mathrm{cmH}_{2} \mathrm{O}$; Group 2, long protocol (LP) recruited with PEEP of $20 \mathrm{cmH}_{2} \mathrm{O}$; and Group 3, control protocol $(\mathrm{CoP})$ ventilated with PEEP of $0 \mathrm{cmH}_{2} \mathrm{O}$. For each maneuver, the central-peripheral temperature gradient (TG), capillary refill time (CRT) and pulse oximetry (SpO2) were evaluated, in four times namely: T1 (Pre-anesthesia), T2 (pre-recruitment), T3 (30 min post-maneuver) and T4 (60 min post-maneuver). In T2, CRT values showed a significant decrease $(\mathrm{p}<0.05)$, with respect to baseline time. Subsequently, in the T3, a significant statistical decrease of the TG for the SP group was evident compared to the other treatments; while in the T4 the CRT presented a significant decrease $(\mathrm{p}<0.05)$ for LP group versus the other treatments. It is suggested that under the conditions presented here, the protocol of ventilation with PEEP increments of up to $20 \mathrm{cmH}_{2} \mathrm{O}$ is the ARM of choice as it does not produce alterations of clinical importance in the microcirculatory parameters evaluated.

Keywords: anesthesia; anesthetic monitoring; mechanical ventilation; pulmonary atelectasis.

\section{INTRODUCCIÓN}

Las maniobras de reclutamiento alveolar (MRA) aplicadas a perros sometidos a anestesia general son de vital importancia, ya que son el blanco de estados patológicos transitorios, como la atelectasia alveolar perioperatoria, la cual, se genera por tres mecanismos patológicos diferentes: compresión, absorción del gas alveolar y pérdida del surfactante (Staffieri et al. 2010; Vargas et al. 2013). Estudios previos evidencian la utilidad de las MRA en caninos sometidos a anestesia general, reflejándose en una mejor ventilación pulmonar, adecuada saturación de oxígeno y un correcto intercambio gaseoso (Staffieri et al. 2010; Canfrán et al. 2012; Ospina et al. 2014; Buriticá et al. 2016); no obstante, la realización de estas maniobras trae consigo un riesgo inherente, al producir altas presiones intratorácicas y transpulmonares, conducentes a una reducción del gasto cardíaco y del volumen sistólico (Monge et al. 2012).

Según Ochagavia et al. (2009), el entendimiento de las alteraciones hemodinámicas generadas por las MRA repercutirá directamente en su rápida identificación y, por lo tanto, en la minimización de eventos adversos; no obstante, sin un mecanismo de monitoreo microcirculatorio invasivo ajustado al contexto veterinario colombiano, lograr esta meta dependerá, como lo afirman Hernández-Gonzales \& Salgado (2016), de la implementación de estrategias menos objetivas, como la evaluación clínica del paciente, una adecuada interpretación de sus parámetros macrocirculatorios, como por ejemplo, la medición del pulso arterial o la evaluación de su producción de orina, lo cual, implicaría el uso de catéteres urinarios, sumado al tiempo y el riesgo de aparición de una infección de tipo hospitalario asociado al catéter (Stull \& Weese, 2015).

Diferentes estrategias de monitoreo microcirculatorio han sido descritas, siendo una de ellas, la valoración del tiempo de relleno capilar, como parámetro fisiológico, útil en la identificación temprana de alteraciones hemodinámicas intraoperatorias en el perro (Silverstein et al. 2014). Un segundo parámetro a realizar es el monitoreo de la oximetría de pulso $\left(\mathrm{SpO}_{2}\right)$, por medio de espectrofotometría infrarroja, que se ha implementado en la valoración de los cambios hemodinámicos microcirculatorios de gatos, anestesiados con isoflurano (Goodnight et al. 2015). Finalmente, la estimación del gradiente de temperatura centro periférico, como medida que denota, de manera subjetiva, el grado de perfusión periférica, con base en la diferencia entre la temperatura central y la temperatura periférica (Réa-Neto et al. 2006; Floriano et al. 2016).

Estas estrategias de monitoreo, se caracterizan por su carácter no invasivo, su fácil consecución y su practicidad para el común de los médicos veterinarios, del contexto colombiano; sin embargo, del conocimiento de los autores, no existe evidencia científica publicada, que permita esclarecer el comportamiento de algunos parámetros de monitoreo microcirculatorio durante la realización de las MRA, establecidas en el presente estudio. Por tal razón, el objetivo de esta investigación fue evaluar el efecto de dos maniobras cíclicas de reclutamiento alveolar sobre diversos parámetros de monitoreo microcirculatorio no invasivo en perros.

\section{MATERIALES Y MÉTODOS}

Se realizó un estudio clínico prospectivo, ciego y aleatorizado, avalado por el comité de Bioética de la Universidad del Tolima, elaborado bajo los lineamientos de las leyes 84 de 1989 y 1774 de 2016 de protección animal y desarrollado en la Clínica de Pequeños Animales de la Universidad del Tolima (CPA-UT), localizada en Ibagué - Tolima, Colombia, a una altura de $1.527 \mathrm{~m}$ s.n.m., una presión barométrica de $657 \mathrm{mmHg}$ y una temperatura promedio de $28,9^{\circ} \mathrm{C}$, durante febrero y julio de 2017.

Animales: 28 perros clínicamente sanos de diferentes razas, con un peso de 25,8 \pm 9,0kg y una edad de 37,7 $\pm 20,3$ meses, sometidos a profilaxis dental, fueron incluidos en el estudio, después de obtener el correspondiente consentimiento informado. Los animales fueron considerados como ASA I, correspondiente a mínimo riesgo anestésico, según clasificación de la Sociedad Americana de Anestesiólogos -ASA-, con base en su historia clínica, examen físico, proteínas plasmáticas totales y cuadro hemático de segunda generación. Todo paciente con alguna anomalía en las pruebas descritas o con signos de enfermedad sistémica o cardio-ventilatoria fueron excluidos del estudio; de igual manera, fenotipos raciales braquicefálicos, agresividad, ansiedad extrema y obesidad mórbida, fueron considerados como factores de exclusión. Como criterios de eliminación fueron considerados asincronía paciente - ventilador o muerte perianestésica. 
Los animales fueron sometidos a un ayuno sólido de $6 \mathrm{~h}$ y líquido de $2 \mathrm{~h}$. La vena cefálica derecha fue canalizada con un catéter intravenoso 20-22 G (Nipro Medical Corporation, Osaka, Japón), por el cual, fue administrado cloruro de sodio al $0,9 \%$ (Cloruro de sodio $0,9 \%$, Gabrica, Bogotá, Colombia), a una velocidad de infusión de $5 \mathrm{~mL} / \mathrm{kg} / \mathrm{h}$, por medio de una bomba de infusión (Bomba de infusión SK-600 I, SK Medical, Shenzhen, China). La medicación preanestésica consistió en $0,3 \mathrm{mg} / \mathrm{kg}$ IM de meloxicam al $0,5 \%$ (Meloxic ${ }^{\circledR}$, Provet, Medellín, Colombia). La inducción anestésica, se realizó con $8 \mathrm{mg} / \mathrm{kg}$ IV de propofol al 1\% (Profol ${ }^{\mathrm{TM}} 1 \%$, Claris injectables limited, Ahmedabad, India).

Acto seguido, se realizó la intubación orotraqueal, con un tubo endotraqueal con sistema de neumotaponamiento, acorde al tamaño del animal. El mantenimiento anestésico, se efectuó con isoflurano (Isoflurano USP, Piramal HealthCare Ltd, Digwal, India), en oxígeno al 100\%, a un flujo de gas fresco de $100 \mathrm{~mL} / \mathrm{kg} / \mathrm{min}$. Inicialmente, la ventilación fue de carácter en un circuito circular. El gas anestésico fue ajustado a una fracción espirada del mismo (EtISO) de 1,5\%. Transcurridos $10 \mathrm{~min}$, se dio inicio a la ventilación mecánica controlada por volumen, por medio de un ventilador mecánico (Wato EX - 65, Mindray Bio-medical electronics co, Ltda, Shenzhen, China), de la siguiente manera: flujo de gas fresco $100 \mathrm{~mL} / \mathrm{kg} / \mathrm{min}$, volumen tidal $8 \mathrm{~mL} / \mathrm{kg}$, relación inspiración:espiración 1:2, pausa inspiratoria del $20 \%$, PEEP $0 \mathrm{cmH}_{2} \mathrm{O}$ y frecuencia respiratoria ajustada, para mantener una fracción espirada de $\mathrm{CO}_{2}$ de $35-45 \mathrm{mmHg}$.

Transcurridos diez minutos de iniciada la ventilación controlada por volumen, se cambió el modo ventilatorio al mandatorio por presión, de la siguiente manera: presión inspiratoria pico $10 \mathrm{cmH}_{2} \mathrm{O}, \mathrm{PEEP} 0 \mathrm{cmH}_{2} \mathrm{O}$ y frecuencia respiratoria $20 \mathrm{ciclos} / \mathrm{min}$. A continuación, los animales fueron asignados al azar, mediante un sistema de aleatorización en línea RANDOM.ORG, en uno de tres grupos: Grupo 1 (Protocolo corto - PC, $n=10$ ), reclutado con PEEP de 0-5-10-5-0 $\mathrm{cmH}_{2} \mathrm{O}$; grupo 2 (Protocolo largo - PL, $\mathrm{n}=9$ ), reclutado con PEEP de 0-5-10-15-20-15-10-5-0 $\mathrm{cmH}_{2} \mathrm{O}$ y grupo 3 (Protocolo control $-\mathrm{Co}, \mathrm{n}=9$ ), reclutado con PEEP de $0 \mathrm{cmH}_{2} \mathrm{O}$. Los incrementos y los decrementos de la presión inspiratoria pico y la PEEP, se realizaron a razón de $5 \mathrm{cmH}_{2} \mathrm{O}$ por cada 5 ciclos ventilatorios, respetando un diferencial de presión de $10 \mathrm{cmH}_{2} \mathrm{O}$, entre ellos. Todas las maniobras fueron realizadas por un mismo profesional, con experiencia en maniobras de reclutamiento pulmonar.

Las variables respuesta fueron: gradiente de temperatura centro periférico (GT), tiempo de relleno capilar (TRC) y oximetría de pulso $\left(\mathrm{SpO}_{2}\right)$. La temperatura central fue valorada mediante medición rectal, por medio de un termómetro digital (MediChek, California, USA) y la temperatura periférica, para hallar el GT, fue valorada sobre la superficie interdigital dorsal del miembro pélvico derecho, por medio de un termómetro infrarrojo, con emisividad ajustada a 0,98 (Termómetro IR FLUKE 568, Fluke Corporation, Washington, USA); el tiempo de relleno capilar (TRC) fue evaluado en la mucosa gingival, tras ejercer una leve presión durante 3 seg y la oximetría de pulso $\left(\mathrm{SpO}_{2}\right)$ fue evaluada mediante sensor de pinza, ubicado en la lengua (iPM12 Vet Monitor, Shenzhen, China).
Los datos obtenidos fueron registrados por un único evaluador en cuatro tiempos, así: T1 (basal en pre-anestesia), T2 (prereclutamiento), T3 (30 min post-maniobra) y T4 (60 min postmaniobra, momento en el que los perros se encontraban incorporados totalmente de la anestesia).

Adicionalmente, los registros de frecuencia cardiaca (FC), frecuencia respiratoria $(\mathrm{FR})$, temperatura esofágica $\left(\mathrm{T}^{\mathrm{T}}\right)$, presión arterial sistólica (PAS), presión arterial diastólica (PAD) y presión arterial media (PAM) fueron evaluados cada 5 min y en cada uno de los tiempos experimentales, por medio de un monitor multiparamétrico (Monitor T8, Mindray Medical International Limited, Shenzhen, China).

Para realizar el análisis estadístico, se empleó un análisis descriptivo exploratorio unidimensional, para hallar medidas de tendencia central, paramétricas (media y DE) o no paramétricas (mediana). La distribución normal de los datos fue verificada por medio de la prueba de Shapiro Wilk. El test de análisis de varianza de una vía (ANOVA) para mediciones repetidas fue empleado para definir la diferencia entre variables. Los análisis no paramétricos fueron realizados mediante la prueba U-Mann Whitney, como post hoc. Un valor de $\mathrm{p}<0,05$ fue considerado como estadísticamente significativo. Los resultados fueron analizados mediante el software estadístico Graph Pad Prism 6.0, California, USA.

\section{RESULTADOS Y DISCUSIÓN}

Del conocimiento de los autores, este es el primer estudio que describe el comportamiento de los parámetros de monitoreo microcirculatorio no invasivo, basados en el gradiente de temperatura centro periférico, el tiempo de relleno capilar y la oximetría de pulso, en perros ventilados con las MRA propuestas. Los resultados de esta investigación evidencian cambios en los parámetros gradiente de temperatura centro periférico y tiempo de relleno capilar, después de 30 y 60 minutos, respectivamente, de haber realizado las MRA, empleadas en este trabajo.

El estudio fue desarrollado sin complicaciones clínicas, a excepción de dos perros que fueron retirados de la investigación, por presentar asincronía paciente-ventilador, los cuales, no fueron tenidos en cuenta en el número $\mathrm{n}$ de animales muestreados.

En la tabla 1, se observan los valores de los parámetros fisiológicos preanestésicos evaluados (T1), los cuales, se encontraron en los límites propios para la especie (Haberman et al. 2006; Réa-Neto et al. 2006; Defarges, 2015; Auckburally, 2016; Thomas \& Lerche, 2017). Adicionalmente, se observa el efecto de la inducción anestésica sobre los parámetros de monitoreo (T2), encontrando cambios estadísticamente significativos $(\mathrm{p}<0,05)$, en los valores de FC, FR, PAS, PAD, PAM y TRC. Dichos valores son similares a los ya reportados por Redondo et al. (2007), para la especie.

El común de los fármacos anestésicos genera un impacto directo sobre la función cardioventilatoria del paciente (Thomas \& Lerche, 2017). El propofol, se relaciona, principalmente, con una disminución de la resistencia vascular periférica y del gasto cardiaco, llevando al 
animal a un estado de hipotensión transitoria dosis dependiente, sin un incremento compensatorio de la frecuencia cardíaca (Berry, 2015; Thomas \& Lerche, 2017). Según lo descrito por Thomas \& Lerche (2017), estos efectos adversos son comunes tras el uso de gases anestésicos, como el isoflurano, exceptuando la FC, que tiende a conservarse o a aumentar, debido al efecto vagolítico inherente del gas (Clarke et al. 2014). Por otra parte, el propofol y el isoflurano, se relacionan con episodios bradipnéicos dependientes de la dosis empleada, según lo afirmado por Clarke et al. (2014) y Steffey et al. (2015), lo que concuerda con los resultados del T2, con una FR más baja frente a la pre-anestésica. A pesar de los cambios descritos, no tuvieron una repercusión clínica importante in situ, por lo que no se empleó acción correctiva.
El TRC es definido como el tiempo que se requiere para retomar la coloración inicial al ejercer una presión en un lecho capilar distal, preferiblemente, despigmentado (Pickard et al. 2011). Con un valor fisiológico inferior a $2 \mathrm{seg}$, este parámetro brinda información sobre el estado de perfusión periférica (Stephenson, 2014). Los cuadros clínicos asociados a hipoperfusión periférica por vasoconstricción tendrán, por consiguiente, valores del TRC incrementados, mientras que aquellos relacionados con vasodilatación, lo tendrán disminuido (Defarges, 2015; Silverstein \& Hopper, 2015); adicionalmente, se ha reportado una fuerte correlación entre un TRC mayor a 2 seg y una saturación venosa de oxígeno $<70 \%$ en niños críticamente enfermos (Bustos \& Padilla, 2014); sin embargo, esta asociación no ha sido demostrada en perros.

Tabla 1. Valor promedio y desviación standard $( \pm \mathrm{SD})$ de los parámetros de monitoreo microcirculatorio no invasivo en el periodo de pre-anestesia (T1) y pre-reclutamiento (T2).

\begin{tabular}{|c|c|c|c|}
\hline Parámetro & Pre-anestesia T1 & Pre-reclutamiento T2 & Significancia $\mathbf{p}$ \\
\hline FC (latidos/minuto) & $94 \pm 21,3$ & $112 \pm 20,6$ & $*$ \\
\hline FR (ventilaciones/minuto) & $35 \pm 13,9$ & $17 \pm 5,8$ & NS \\
\hline $\left.\mathbf{T R}^{\circ} \mathbf{C}\right)$ & $38,6 \pm 0,5$ & $38,4 \pm 0,5$ & NS \\
\hline $\mathbf{G T}\left({ }^{\circ} \mathbf{C}\right)$ & $4,9 \pm 1,3$ & $4,9 \pm 1,6$ & $*$ \\
\hline PAS (mmHg) & $145 \pm 30,5$ & $103 \pm 15,2$ & $*$ \\
\hline PAD (mmHg) & $89 \pm 23,3$ & $49 \pm 13,3$ & NS \\
\hline PAM (mmHg) & $105 \pm 23,1$ & $65 \pm 11,6$ & $*$ \\
\hline SpO $(\mathbf{m}$ ) & $93,4 \pm 3,4$ & $94,7 \pm 2,8$ & $1 \pm 0,4$ \\
\hline TRC (segundos) & $2 \pm 0,5$ & & $*$ \\
\hline
\end{tabular}

Frecuencia cardiaca (FC), frecuencia respiratoria (FR) (ventilaciones/minuto), temperatura rectal $(\mathrm{TR})\left({ }^{\circ} \mathrm{C}\right)$, gradiente de temperatura centro periférico $(\mathrm{GT})\left({ }^{\circ} \mathrm{C}\right)$, presión arterial sistólica (PAS) (mmHg), presión arterial diastólica (PAD) (mmHg), presión arterial media (PAM) ( $\mathrm{mmHg}$ ), oximetría de pulso (SpO2) (\%) y tiempo de relleno capilar (TRC) (segundos). Significancia estadística $\mathrm{p}<0,05^{*}$.

La interpretación del TRC, se puede sesgar por factores, como la edad del perro, la temperatura corporal, la luz ambiental, la presión de aplicación digital y el grado de variabilidad intra e interobservador (Pickard et al. 2011); no obstante, estos factores se tuvieron en cuenta al momento de plantear el diseño metodológico del estudio, logrando homogenizar las variables, mediante el uso de animales adultos jóvenes, sin evidencia de hipo o hipertermia trasanestésica o presión gingival controlada en tiempo, lo cual, permitió minimizar su aparición. Floriano et al. (2016) estudiaron el efecto del isoflurano a diferentes concentraciones alveolares sobre la función microcirculatoria en perros y encontraron que el efecto vasodilatador de este gas anestésico tiene una relación benéfica sobre la perfusión periférica, ya que los valores del TRC se mantienen estables o pueden disminuir al momento de la valoración, hallazgos que concuerdan con resultados de este estudio, puesto que en el periodo anestésico el TRC fue menor frente al periodo preanestésico.
De igual manera, se ha planteado que la ventilación mecánica altera el funcionamiento hemodinámico, debido a los cambios de la presión intratorácica que ella supone, lo cual, se evidencia con disminuciones del retorno venoso, gasto cardiaco y volumen sistólico, según lo afirmado por Ochagavia et al. (2009); sin embargo, en el T3 se puede observar que el TRC, posiblemente, no fue influenciado por estos aspectos, hallazgos de los que se infiere pueden ser el resultado del efecto vasodilatador del isoflurano, como fue anteriormente mencionado.

Monge et al. (2012) describieron los cambios hemodinámicos ocasionados por una MRA cíclica en humanos, demostrando que se producen cambios importantes en el gasto cardiaco y el volumen sistólico; sin embargo, estas alteraciones desaparecen conforme termina la MRA, con lo cual, se descartaría cualquier efecto de los protocolos de ventilación sobre los resultados del T3 y T4, de los dos protocolos de ventilación (PC y PL) (Tabla 2). Finalmente, se 
observa una disminución significativa del TRC en el T4 del PL frente a los demás $(\mathrm{p}=0,0094)$, destacando que, en ese momento, los animales ya se habían incorporado de la anestesia y teniendo como base el planteamiento de Monge et al. (2012), anteriormente descrito, se infiere que esta disminución se relacionó con la ansiedad que mostraron los perros en la recuperación anestésica

El GT, se ha sugerido como herramienta clínica para monitorear rápidamente la perfusión periférica de manera no invasiva (Lima \& Bakker, 2005). Este parámetro evalúa subjetivamente la perfusión periférica con base en la diferencia de la temperatura central y la temperatura periférica, siendo normales los valores comprendidos entre 3 y $7^{\circ} \mathrm{C}$. De esta forma, cualquier alteración de la perfusión periférica se reflejará en un GT superior a $7^{\circ} \mathrm{C}$ (Réa-Neto et al. 2006). Los valores del GT para el T1 y T2 estuvieron dentro de los limites clínicos, sin diferencia entre ellos (Tabla 1); no obstante, se observa una reducción significativa en el GT para el T3 del PC respecto a los demás tratamientos $(\mathrm{p}=0,0429)$, con valores similares a los reportados por Floriano et al. (2016), a los 20min de anestesia con isoflurano. Partiendo de la premisa de que un alto flujo sanguíneo facilita la transferencia de calor del compartimento central al compartimento periférico, según lo reportado por Lima \& Bakker (2005), el efecto vasodilatador del isoflurano haría disminuir el valor del GT.

De igual manera, la ventilación mecánica y las MRA pueden afectar, de manera conjunta, los marcadores macrocirculatorios, como, por ejemplo, el gasto cardíaco tras su efecto sobre la presión intratorácica, según lo afirmado por Ochagavia et al. (2009) y Monge et al. (2012), lo cual, pudo tener un efecto sobre la circulación periférica en el presente estudio, lo que explicaría la diferencia entre los valores de GT en el T3, para los diferentes tratamientos.

Por último, se encontró un aumento no estadísticamente significativo en los valores promedio del GT en el T4 de todos los tratamientos. Redondo et al. (2012) demostraron que luego de 45min de anestesia inhalatoria bajo condiciones de ambiente controlado, se produce una reducción de $2^{\circ} \mathrm{C}$ de la temperatura central, lo que podría estar igualmente asociado a una disminución del flujo sanguíneo al compartimento periférico (Lima \& Bakker, 2005). Teniendo en cuenta lo anterior, es posible que los resultados del T4 se hayan visto influenciados por el efecto de la anestesia y del flujo del gas fresco, el cual, al ser elevado facilita la pérdida de calor por la ventilación (Lachin, 2012a; 2012b). Todo esto, posiblemente, influyó para que el GT del T4, en promedio, fuera superior frente al T3.

La oximetría de pulso hace referencia a la medición porcentual del oxígeno que se encuentra unido a la molécula de hemoglobina, cuyo valor fisiológico es $\geq 94 \%$ (Auckburally, 2016). Los resultados obtenidos estuvieron dentro los rangos normales en los momentos evaluados (Tablas 1 y 2); no obstante, se observó un incremento de esta en los tiempos experimentales T2 y T3, en los que el paciente estuvo bajo la influencia de la ventilación con una fracción inspirada de oxígeno $\left(\mathrm{FiO}_{2}\right)$ de 1,0. Estos hallazgos concuerdan con los resultados descritos por Buriticá et al. (2016), quienes demostraron que una $\mathrm{FiO}_{2}$ de 1,0 genera un incremento de la

Tabla 2. Valor promedio y desviación standard $( \pm S D)$ de los parámetros de monitoreo microcirculatorio no invasivo, registrados a los 30min post-reclutamiento (T3) y 60min post-reclutamiento (T4), en perros ventilados con dos diferentes maniobras cíclicas de reclutamiento alveolar.

\begin{tabular}{|c|c|c|c|c|c|c|}
\hline \multirow[t]{2}{*}{ Parámetros } & \multicolumn{3}{|c|}{$\begin{array}{c}\text { 30min post-reclutamiento } \\
\text { T3 }\end{array}$} & \multicolumn{3}{|c|}{$\begin{array}{c}60 \mathrm{~min} \text { post-reclutamiento } \\
\mathrm{T} 4\end{array}$} \\
\hline & $P C(n=10)$ & PL $(n=9)$ & PCo $(n=9)$ & $P C(n=10)$ & PL $(n=9)$ & PCo $(n=9)$ \\
\hline FC (latidos/minuto) & $102 \pm 14,6$ & $105 \pm 11,0$ & $99 \pm 13,2$ & $106 \pm 16,3$ & $100 \pm 14,3$ & $108 \pm 13,9$ \\
\hline FR (ventilaciones/minuto) & $14 \pm 3,9$ & $17 \pm 4,2$ & $18 \pm 4,4$ & $28 \pm 12,7$ & $33 \pm 10,2$ & $30 \pm 9,8$ \\
\hline $\operatorname{TR}\left({ }^{\circ} \mathrm{C}\right)$ & $38,1 \pm 1,5$ & $38,2 \pm 0,6$ & $37,4 \pm 0,4$ & $38,0 \pm 0,7$ & $38,2 \pm 0,8$ & $37,5 \pm 0,6$ \\
\hline PAS (mmHg) & $113 \pm 16,8$ & $114 \pm 22,5$ & $107 \pm 24,7$ & $139 \pm 26,9$ & $134 \pm 20,0$ & $128 \pm 32,2$ \\
\hline $\mathrm{PAD}(\mathrm{mmHg})$ & $53 \pm 15,2$ & $58 \pm 19,1$ & $51 \pm 22,2$ & $85 \pm 20,5$ & $82 \pm 26,1$ & $84 \pm 11,6$ \\
\hline PAM (mmHg) & $69 \pm 13,2$ & $76 \pm 18,7$ & $73 \pm 23,6$ & $99 \pm 20,5$ & $101 \pm 23,9$ & $99 \pm 14,7$ \\
\hline SpO2 (\%) & $100 \pm 2,4$ & $96 \pm 2,1$ & $95 \pm 2,3$ & $96 \pm 5,5$ & $94 \pm 1,9$ & $94 \pm 2,6$ \\
\hline
\end{tabular}

Protocolo corto (PC), protocolo largo (PL), protocolo control (PCo), Frecuencia cardiaca (FC) (latidos/minuto), frecuencia respiratoria (FR) (ventilaciones/minuto), temperatura rectal $(\mathrm{TR})\left({ }^{\circ} \mathrm{C}\right)$, gradiente de temperatura centro periférico $(\mathrm{GT})\left({ }^{\circ} \mathrm{C}\right)$, presión arterial sistólica (PAS) (mmHg), presión arterial diastólica (PAD) ( $\mathrm{mmHg})$, presión arterial media (PAM) (mmHg), oximetría de pulso (SpO2) (\%) y tiempo de relleno capilar (TRC) (segundos). Significancia estadística $\mathrm{p}<0,05^{*}$. 
presión arterial de oxígeno en promedio de $500 \mathrm{mmHg}$. En ese orden de ideas, al ser la $\mathrm{SpO}_{2}$ una variable dependiente de los niveles de oxígeno en sangre arterial, se esperaría que su valor se incrementara durante el periodo anestésico. Por otra parte, la $\mathrm{SpO}_{2}$ se relaciona directamente con el grado de perfusión tisular, de tal forma que, en estados de vasoconstricción, la lectura no es precisa (Auckburally, 2016). Contrario a esto y de acuerdo a lo afirmado por Floriano et al. (2016), la vasodilatación derivada del isoflurano al mejorar la perfusión periférica facilitaría una óptima medición de dicho parámetro; sin embargo, ha de considerarse el hecho que, en el presente estudio, no fue posible hacer una medición del valor de presión arterial de oxígeno $(\mathrm{PaO} 2)$, con el fin de correlacionar los resultados encontrados.

Por otra parte, la fluidoterapia hace parte fundamental del cuidado transanestésico como suplemento hemodinámico, minimizando la incidencia de cuadros hipotensivos, los cuales, afectarían la perfusión periférica (Fantoni \& Shih, 2017); sin embargo, Silverstein et al. (2014) demostraron que emplear fluidoterapia liberal $(20 \mathrm{~mL} /$ $\mathrm{kg} / \mathrm{h}$ ) frente a una $100 \%$ restrictiva $(0 \mathrm{~mL} / \mathrm{kg} / \mathrm{h})$, no genera cambios microcirculatorios significativos en perras sometidas a ovariohisterectomía electiva. La fluidoterapia empleada en este estudio $(5 \mathrm{~mL} / \mathrm{kg} / \mathrm{h})$ permite inferir que, desde los parámetros de monitoreo microcirculatorio no invasivo evaluados, es benéfica para en el transanestésico de perros sometidos a las MRA, acá descritas.

Como alternativa a los métodos de monitoreo empleados en esta investigación, se han planteado aquellos que comprenden un monitoreo óptico, como lo es el campo oscuro lateral o la imagen polarizada ortogonal espectral, según lo descrito por Silverstein et al. (2009) y Goodnight et al. (2015), que permiten visualizar en tiempo real el reclutamiento de la microcirculación; no obstante, estos no permiten evaluar la microcirculación desde un punto de vista sistémico, quedando limitada la evaluación únicamente a la cavidad oral; además, dicha tecnología reviste un costo significativo que limita su aplicabilidad. Otra alternativa es la medición de la saturación venosa central de oxígeno, que brinda una perspectiva sobre el estado microcirculatorio general del organismo, pero es un pobre indicador de alteraciones tisulares específicas; además, demanda un grado de experticia, ya que para su medición requiere ubicar un catéter a nivel de la arteria pulmonar (Floriano et al. 2016).

Tras la realización del presente estudio es importante considerar que no fue posible estimar el reclutamiento de la microcirculación de una manera objetiva, debido al carácter de no invasividad de la investigación. Esto podría ser tenido en cuenta para la realización de trabajos futuros, que incluyan diversas estrategias invasivas, para hacer una interpretación más objetiva de los resultados.

Emplear protocolos anestésicos carentes de tranquilización puede sesgar los resultados de algunos parámetros microhemodinámicos; por tal razón, se sugiere evaluar el efecto de diferentes tranquilizantes sobre los parámetros de monitoreo microcirculatorio, acá estudiados.

Los resultados del presente estudio permiten concluir que la realización de maniobras de reclutamiento alveolar cíclicas con incrementos de la presión tele-espiratoria de hasta $20 \mathrm{cmH}_{2} \mathrm{O}$, no generan alteraciones clínicas significativas en los parámetros microcirculatorios no invasivos evaluados, por lo cual, se sugiere la implementación de la maniobra de reclutamiento con PEEP de $20 \mathrm{cmH}_{2} \mathrm{O}$ en caninos, para revertir los focos atelectásicos pulmonares perianestésicos. Por su parte, la relevancia clínica del presente estudio, se basa en la aplicabilidad de herramientas de monitorización comunes para el clínico en ejercicio, como lo son el gradiente de temperatura centro periférico (GT), el tiempo de relleno capilar (TRC) y la oximetría de pulso $\left(\mathrm{SpO}_{2}\right)$ en el perro anestesiado o sometido a maniobras de reclutamiento alveolar.

Conflictos de intereses: El manuscrito fue preparado y revisado con la participación de todos los autores, quienes declaramos que no existe conflicto de intereses que ponga en riesgo la validez de los resultados presentados. Financiación: Este estudio fue financiado por la Oficina de Investigaciones y Desarrollo Científico de la Universidad del Tolima.

\section{REFERENCIAS}

1. AUCKBURALLY, A. 2016. Pulse oximetry and oxygenation assessment in small animal practice. Vet. Rec. (Reino Unido). 38(2):50-58. http://dx.doi.org/10.1136/inp.i185

2. BERRY, S.H. 2015. Injectable anesthetics. En: Grimm, K.A.; Lamont, L.A.; Tranquilli, W.J.; Greene, S.A.; Robertson, S.A. (eds). Veterinary Anesthesia and Analgeisa - The Fifth Edition of Lumb and Jones. Ed. Wiley-Blackwell (Iowa). p.277-296.

3. BURITICÁ, E.F.; ECHEVERRY, D.F.; OSPINA, D.A. 2016. Efecto de dos maniobras manuales cíclicas de reclutamiento alveolar sobre gases sanguíneos en perros anestesiados. Rev. U.D.C.A Act. \& Div. Cient. (Colombia). 19(1):161-168. https://doi.org/10.31910/rudca.v19. n1.2016.123

4. BUSTOS, R.; PADILLA, O. 2014. El tiempo de llene capilar prolongado es predictor de una saturación venosa central de oxígeno disminuida. Rev. Chil. Pediatr. 85(5):539-545. http://dx.doi.org/10.4067/S0370-41062014000500003

5. CANFRÁN, S.; GÓMEZ DE SEGURA, I.; CEDIEL, R.; GARCÍA-FERNÁNDEZ, J. 2012. Effects of a stepwise lung recruitment manoeuvre and positive end-expiratory pressure on lung compliance and arterial blood oxygenation in healthy dogs. Vet.J. (Reino Unido). 194(1):89-93. http:/ / doi.org/10.1016/j.tvj1.2012.02.021

6. CLARKE, K.W.; TRIM, C.M.; HALL, L.W. 2014. Veterinary anaesthesia. Ed. Elsevier (Estados Unidos). 694p.

7. DEFARGES, A. 2015. The physical examination. Clinician's Brief (Estados Unidos). Disponible desde internet en: http://www.cliniciansbrief.com/sites/default/files/ 
attachments/ProP_Physical\%20Examination.pdf (con acceso 13/04/2017)

8. FANTONI, D.; SHIH, A.C. 2017. Perioperative fluid therapy. Vet. Clin. North Am. Small Anim. Pract. (Estados Unidos). $47(2): 423-434$.

9. FLORIANO, B.P.; WAGATSUMA, J.T.; FERREIRA, J.Z.; ABIMUSSI, C.J.; MENEGHETI, T.M.; SANTOS, P.S.; OLIVA, V.N. 2016. Effects on indicators of tissue perfusion in dogs anesthetized with isoflurane at two multiples of the minimum alveolar concentration. Am. J. Vet. Res. (Estados Unidos). 77(1):24-31. https://doi. org/10.2460/ajvr.77.1.24

10. GOODNIGHT, M.E.; COOPER, E.S.; BUTLER, A.L. 2015. Assessment of microcirculatory perfusión in healthy anesthetized cats undergoing ovariohisterectomy using sidestream dark field microscopy. J. Vet. Emerg. Crit. Care (Reino Unido). 25(3):349-357. https://doi.org/10.1111/ vec. 12296

11. HABERMAN, C.E.; KANG, C.W.; MORGAN, J.D.; BROWN, S.A. 2006. Evaluation of oscillometric and Doppler ultrasonic methods of indirect blood pressure estimation in conscious dogs. Can. J. Vet. Res. 70(3):211-217.

12. HERNÁNDEZ-GONZALES, G.; SALGADO, J. 2016. Monitorización de la perfusión tisular en el paciente críticamente enfermo. Rev. Cient. Cienc. Méd. (Bolivia). 19(2):43-47.

13. LACHIN, A. 2012a. Ipotermia accidentale intra - e perioperatoria. Anestesia cane, gatto e animali non convenzionali. Ed. Elsevier (Milán). p.285-291.

14. LACHIN, A. 2012b. Sistemi respiratori. Anestesia cane, gatto e animali non convenzionali. Ed. Elsevier (Milán). p.221239.

15. LIMA, A.; BAKKER, J. 2005. Noninvasive monitoring of peripheral perfusion. Intensive Care Med. (Alemania). 31(10):1316-1326. https://doi.org/10.1007/s00134-005$2790-2$

16. MONGE, M.I.; GIL, A.; GRACIA, M.; DÍAZ, J.C. 2012. Cambios respiratorios y hemodinámicos durante una maniobra de reclutamiento pulmonar mediante incrementos y decrementos progresivos de PEEP. Med. Intensiva (España). 36(2):77-88.

17. OCHAGAVIA, A.; BLANCH, L.; LÓPEZ-AGUILAR, J. 2009. Utilidad de las maniobras de reclutamiento (contra). Med. Intensiva (España). 33(3):139-143.
18. OSPINA, D.A.; BURITICÁ, E.F.; ECHEVERRY, D.F.; VILLANUEVA, C.L.; RONDÓN, I.S. 2014. Maniobras de reclutamiento alveolar en el control de la atelectasia pulmonar. Veterinaria y Zootecnía (Colombia). 8(1):17-34.

19. PICKARD, A.; KARLEN, W.; ANSERMINO, J.M. 2011. Capillary refill time: is it still a useful clinical sign? Anesth. Analg. (Estados Unidos). 113(1):120-123. https://doi. org/10.1213/ANE.0b013e31821569f9

20. RÉA-NETO, A.; REZENDE, E.; MENDES, C.L.; DAVID, C.M.; DIAS, F.S.; SCHETTINO, G.; AJEJE, S.M. 2006. Consenso brasileiro de monitorização e suporte hemodinâmico - Parte IV: Monitorização da perfusão tecidual. Rev. Bras. Ter. Intensiva. 18(2):154-160. https:// doi.org/10.1590/S0103-507X2006000200009

21. REDONDO, J.I.; RUBIO, M.; SOLER, G.; SERRA, I.; SOLER, C.; GÓMEZ-VILLAMANDOS, R.J. 2007. Normal values and incidence of cardiorespiratory complications in dogs during general anaesthesia. A review of 1281 cases. J. Vet. Med. A Physiol. Pathol. Clin. Med. (Alemania). 54(9):470477. https://doi.org/10.1111/j.1439-0442.2007.00987.x

22. REDONDO, J.I.; SUESTA, P.; SERRA, I.; SOLER, C.; SOLER, G.; GIL, L.; GÓMEZ-VILLAMANDOS, R.J. 2012. Retrospective study of the prevalence of postanaesthetic hypothermia in dogs. Vet. Rec. (Reino Unido). 171(15):374. https://doi.org/10.1136/vr.100476

23. SILVERSTEIN, D.C.; HOPPER, K. 2015. Small Animal Critical Care Medicine. 2da edición. Ed. Elsevier (Estados Unidos). 1130p.

24. SILVERSTEIN, D.C.; PRUET'T-SARATAN II, A.; DROBATZ, K.J. 2009. Measurements of microvascular perfusion in healthy anesthetized dogs using orthogonal polarization spectral imaging. J. Vet. Emerg. Crit. Care (Estados Unidos). 19(6):579-586. https://doi.org/10.1111/j.14764431.2009.00488.x

25. SILVERSTEIN, D.C.; COZZI, E.M.; HOPKINS, A.S.; KEEFE, T.H. 2014. Microcirculatory effects of intravenous fluid administration in anesthetized dogs undergoing elective ovariohysterectomy. Am. J. Vet. Res. (Estados Unidos). 75(9):809-817. https://doi.org/10.2460/ajvr.75.9.809

26. STAFFIERI, F.; DE MONTE, V.; DE MARZO, C.; SCRASCIA, F.; CROVACE, A. 2010. Alveolar recruiting maneuver in dogs under general anesthesia: effects on alveolar ventilation, gas exchange, and respiratory mechanics. Vet. Res Commun. (Holanda). 34(1):S131-S134. https://doi. org/10.1007/s11259-010-9405-2

27. STEFFEY, E.P.; MAMA, K.R.; BROSNAN, R.J. 2015. Inhalation anesthetics. En: Grimm, K.A.; Lamont, L.A.; 
Tranquilli, W.J.; GREENE, S.A.; Robertson, S.A. (eds) Veterinary Anesthesia and Analgeisa - The fifth edition of Lumb and Jones. Ed. Wiley-Blackwell (Iowa). p.297-331.

28. STEPHENSON, R.E. 2014. Generalidades sobre la función cardiovascular. En: Klein, B.G. (ed). Cunningham Fisiología Veterinaria. Ed. Elsevier (Barcelona). p.158-170.

29. STULL, J.W.; WEESE, J.S. 2015. Hospital-associated infections in small animal practice. Vet. Clin. North. Am. Small Anim. Pract. (Estados Unidos). 45(2):217-233. https:// doi.org/10.1016/j.cvsm.2014.11.009
30. THOMAS, J.A.; LERCHE, P. 2017. Anesthesia and analgesia for veterinary technicians. Ed. Elsevier (Estados Unidos). $445 \mathrm{p}$.

31. VARGAS, M.; BRUNETTI, L.; PELOSI, P. 2013. Protective mechanical ventilation during general anaesthesia. Trends in anesthesia and critical care (Holanda). 3(2):77-81. https://doi.org/10.1016/j.tacc.2012.11.003 